\title{
The Radix Entomolaris and Paramolaris: Case Series of Two Cases and a Short Review
}

\author{
Neelam Mittal, Anoop Mohandas \\ Department of Conservative Dentistry and Endodontics, Faculty of Dental Sciences, Institute of Medical Sciences, Banaras Hindu \\ University, Varanasi, Uttar Pradesh, India
}

Email for correspondence: dr.neelammittal@gmail.com

\begin{abstract}
Endodontists usually come across various anatomical variations, particularly in permanent molars. A thorough knowledge of this aberrant morphology is essential for successful endodontic therapy. Permanent mandibular molars are usually two rooted with two mesial canals and one or two distal canals. Radix entomolaris, an anatomical variant in permanent mandibular molar, is an extra root present on the distolingual position, first described by Carabelli. If the extra root is on buccal aspect, it is termed as radix paramolaris. Even though considered to be an Asiatic trait, its prevalence is low in Indian population. However, its awareness and identification is inevitable to achieve endodontic success.
\end{abstract}

Key words: Endodontic therapy, radix entomolaris, radix paramolaris

\section{INTRODUCTION}

Endodontic therapy primarily aims at the elimination of microbes and tissue debris from the infected root canals by thorough biomechanical preparation followed by three-dimensional obturation with a hermetic seal to prevent further reinfection. Missing additional roots/canals are one of the frequent causes of failure of non-surgical root canal therapy. Mandibular first molars are considered to be most frequently involved tooth in endodontic practice as they erupt early and are known to display several anatomic variations. Hence, an in-depth knowledge of the aberrant morphology should be obtained by the clinician for the successful outcome of endodontic therapy ${ }^{[1,2]}$

The mandibular first molars are usually two rooted with two mesial and one distal canal. ${ }^{[1,2]}$ In majority of cases, mesial root has two canals, ending in two distinct apical foramen or sometimes

\section{Quick Response Code Article Info:}

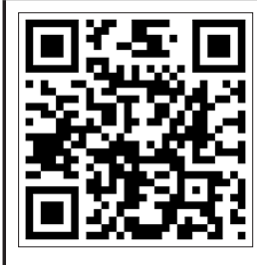

doi: 10.5866/2017.9.10235

Received: 05-12-2017

Revised: $28-12-2018$

Accepted: 05-01-2018

Available Online: 15-02-2018, 2017 (www.

nacd.in)@ NAD, 2017 - All rights reserved they merge at the root top to end in one foramen. Distal root usually presents an oval kidney-shaped single canal, although two canals may be present with narrow and round orifices. ${ }^{[3]}$ However, FabraCampus and Bond reported the presence of three mesial canals while Stroner reported three distal canals. ${ }^{[4-7]}$ Like the canals, number of roots may also vary. In 1844, Carabelli first mentioned an extra root on the distolingual position of permanent mandibular first molar and termed radix entomolaris (RE). ${ }^{[8]}$ If the extra root is on buccal aspect, it is referred as radix paramolaris (RP). ${ }^{[9]}$ The reported prevalence of RE significantly differs with races ranging from 0 to $33.1 \%{ }^{[10]}$ In Indian population, occurrence of $\mathrm{RE}$ and $\mathrm{RP}$ is $<5 \%$ and not routinely observed. Hence, knowledge of the extra roots and canals and radiographs taken at different angulations is essential for delivering a successful endodontic treatment.

\section{CASE REPORTS}

\section{Case 1}

A 25-year-old female patient reported to the department of conservative dentistry and endodontics with pain on the right lower back region which was intermittent for last 2 weeks. The patient reported a history of endodontic therapy done on 46 and 47, 4 months back. The patient's medical 
history was non-significant. Clinical examination revealed amalgam restoration on 46 and 47 , and both were mildly tender on percussion. Radiograph [Figure 1a] revealed incomplete obturation on both 46 and 47 and an extra root on 46. A diagnosis of previously treated tooth with symptomatic apical periodontitis on 46 and 47 was made. Non-surgical retreatment of 46 and 47 was planned.

The patient was given local anesthesia of $2 \%$ lignocaine with 1:80,000 adrenaline. Both 46 and 47 were isolated with rubber dam (GDC India). The amalgam restoration of 46 was removed and access gained into the pulp chamber. The two mesial canals were not obturated and gutta-percha was found on distal canal. An extra canal with GP on it was found buccally between the mesial canals and distal canal. Access opening was little bit modified to become roughly trapezoidal so that there would be an easy access to extra canal of RP. GP was removed from both the canals. The extra canal was approached from the lingual aspect of the crown and patency of the canal was made with 15 number K-file (Dentsply Maillefer Company, USA). Access opening was done on 47 also. Two mesial and one distal canal were located and GP was removed. Working length was determined using electronic apex locator Root ZX (J. Morita MFG. Corporation, Kyoto, Japan) which was confirmed using periapical radiograph [Figure 1b]. The canals were cleaned and prepared using hand K-files (Dentsply Maillefer Company, USA) on stepback technique. $2.5 \%$ sodium hypochlorite was used an irrigant. The intracanal medicament of calcium hydroxide (Prime Dental Products, Thane) was given for 1 week. After 1 week, teeth were asymptomatic. Root canals were reaccessed; intracanal medicament was removed and dried using paper points. Master cone radiograph [Figure 1c] was taken to confirm the suitability of gutta-percha to canals. The canals were obturated with selected master gutta-percha cones, accessory cones, and zinc oxide-eugenol sealer using lateral condensation technique. A temporary restoration was given with Cavitemp. After 1 week, post-endodontic restoration was done on 46 and 47 with amalgam [Figure 1d].

\section{Case 2}

A26-year-old male reported to the department of conservative dentistry and endodontics with pain in relation to the lower right back region for last 3 days. The patient medical history was not significant. Clinical examination revealed the presence of deep distoocclusal caries on right mandibular first molar. Thermal test showed positive and prolonged response. Periapical radiograph [Figure 2a] revealed radiolucency overlapping the pulp chamber from distoocclusal aspect. It also suggests an extra
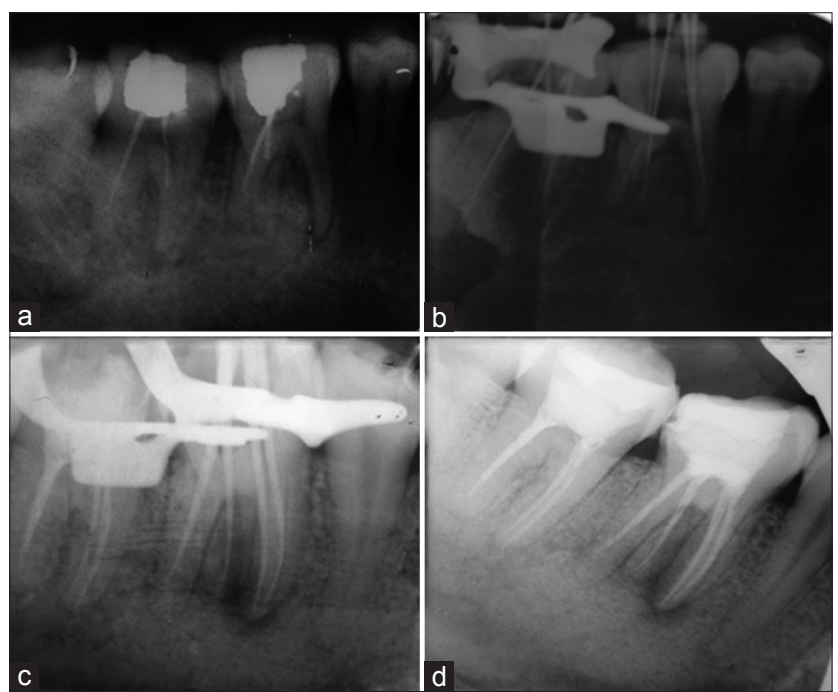

Figure 1: (a) Pre-operative radiograph reveals incomplete obturation in 46 and 47 and an extra root in 46 , (b) radiograph showing working length determination using electronic apex locator Root Zx, (c) radiograph showing master cone suitability of gutta-percha to canals, (d) post-operative radiograph showing endodontic restoration in 46 and 47 with amalgam
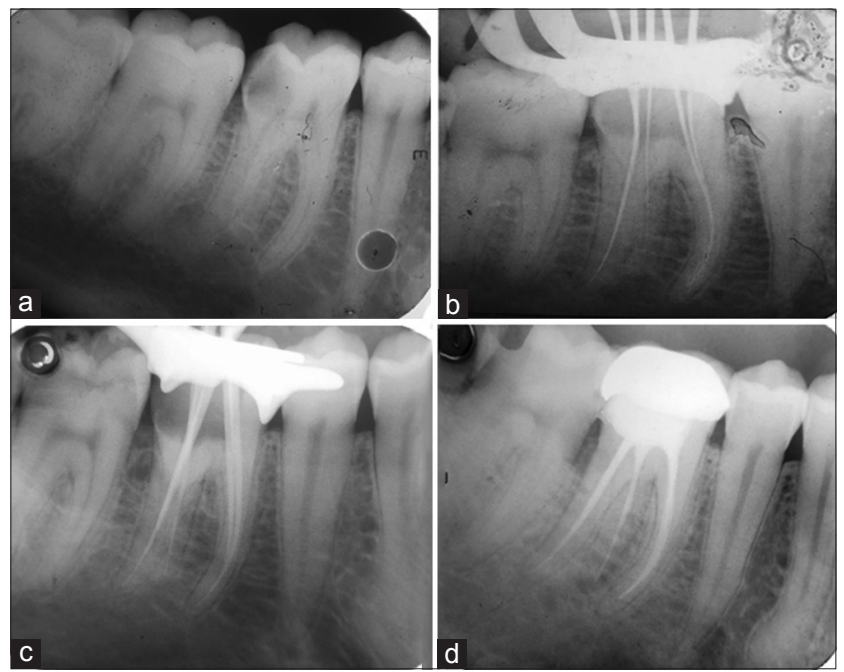

Figure 2: (a) Pre-operative radiograph showing periapical radiolucency overlapping the pulp chamber from distoocclusal aspect. It also suggests an extra root toward the distal root complex, (b) radiograph showing working length determination using electronic apex locator Root ZX, (c) radiograph showing

master cone suitability of gutta-percha to canals, (d) postoperative radiograph showing endodontic restoration in 46 with amalgam 
root toward the distal root complex. Diagnosis of symptomatic irreversible pulpitis was confirmed and non-surgical endodontic therapy was planned.

Local anesthetic of $2 \%$ lignocaine with 1:80,000 adrenaline is administrated and conventional endodontic access was made under rubber dam (GDC India) isolation. On deroofing of pulp chamber, we found two mesial canals and two distal canals. On modifying the access cavity toward the distolingual side, an extra canal was found suggestive of RE. The patency of canals was made with 10 number K-file (Dentsply Maillefer Company, USA). Working length was determined using electronic apex locator Root ZX (J. Morita MFG. Corporation, Kyoto, Japan) and confirmed radiographically [Figure 2b]. Biochemical preparation was done with Rotary Hyflex CM file system using crown-down technique. $2.5 \%$ sodium hypochlorite was used as irrigant and calcium hydroxide as interappointment medicament. The patient was recalled after 1 week and obturation was done with gutta-percha and zinc oxide-eugenol sealer using lateral condensation technique [Figure 2c and d]. Cavitemp was given as temporary restoration. 1 week after, permanent restoration was done with silver amalgam.

\section{DISCUSSION}

The etiology of RE is still unclear. The prevalence of $\mathrm{RE}$ ranges from 0 to $33.1 \%$ differing significantly between races. The prevalence in high among mongoloid group with $>30 \%$ and considered to be eumorphic root morphology. ${ }^{[11-13]}$ In African, Eurasian, Caucasian, and Indian population, it is considered as dysmorphic root morphology with a low prevalence of $<5 \%$. ${ }^{[12-13]}$ In dysmorphic roots, it occurs due to external factors during odontogenesis or attribution to atavic gene or polygenic system. ${ }^{[11,13]}$ The more pronounced phenotypic manifestation of a particular gene under the influence of racial and genetic factors may be the reason in eumorphic roots. ${ }^{[14,15]}$ Slight male predilection is reported in some studies (16) and bilateral occurrence ranges from 37.14 to $67 \%$. ${ }^{[16-18]}$

Bolk reported the occurrence of RP but occurs less frequently than RE. ${ }^{[19]}$ According to Visser, the prevalence of RP was $0 \%$ for mandibular first molars, $0.5 \%$ for second molars, and $2 \%$ for third molars. ${ }^{[20]}$

\section{Classification}

Carlsen and Alexandersen classified RE into four different types based on the location of its cervical part ${ }^{[9]}$ :
1. Type A: The RE is located lingually to the distal root complex which has two cone-shaped macrostructures.

2. Type B: The RE is located lingually to the distal root complex which has one cone-shaped macrostructures.

3. Type $\mathrm{C}$ : The $\mathrm{RE}$ is located lingually to the mesial root complex.

4. Type AC: The RE is located lingually between the mesial a distal root complexes.

De Moor et al. classified RE based on the curvature of the root or root canal ${ }^{[21]}$ :

1. Type 1: A straight root or root canal.

2. Type 2: A curved coronal third which becomes straighter in the middle and apical third.

3. Type 3: An initial curve in the coronal third with a second buccally oriented curve which begins in the middle or apical third.

Song et al. further added two more newly defined variants of $\mathrm{RE}^{[22]}$

1. Small type: Length shorter than half of the length of the distobuccal root.

2. Conical type: Smaller than the small type and having no root canal within it.

\section{Classification of RP}

Carlsen and Alexandersen (1990) classified RP into two different types ${ }^{[9]}$ :

1. Type A: Cervical part is located on the mesial root complex.

2. Type B: Cervical part is located centrally, between the mesial and distal root complexes.

\section{Clinical implications}

Since RE and RP are rare in Indian population, missing of the extra root can happen as the first case reported here. Hence, an accurate diagnosis of these supernumerary roots can avoid complications is the endodontic procedures. A thorough inspection of the pre-operative radiograph and interpretation of particular marks such as unclear view or outline of distal or mesial root contour of root canal can suggest the presence of radix cases. In such cases, additional radiograph taken from different horizontal projections (20 from mesial/distal) reveal information on additional root. ${ }^{[20,23]}$ In cases of RE and RP, the access opening has to be modified to more rectangular or trapezoidal form. The use of magnifying loupes or dental microscope will be helpful. The dentinal map on the purple floor usually 
helps in getting precise location of these extra roots. Most of the times RE and RP have serve root inclination or curvature, particularly in apical third and can lead to procedural errors such as ledges, transportation, and instrument separation. Hence, initial canal exploration with small files, use of $\mathrm{NiTi}$ files, and creating a glide path before biomechanical preparation tooth has to be considered.

In other specialties, considerations include $\mathrm{RE}$ and RP may pose difficulty during extraction and tooth movement may be difficult in orthodontic procedures.

\section{CONCLUSION}

The clinicians should always be aware of unusual root morphologies such as extra canals and roots while treating mandibular molars. An initial diagnosis of $\mathrm{RE}$ and $\mathrm{RP}$ is of utmost importance to facilitate successful endodontic therapy. Radiographs taken at different horizontal angles may help in the diagnosis. A careful clinical approach is needed in cases of RE and RP to give a successful result without any procedural complications.

\section{REFERENCES}

1. Barker BC, Parson KC, Mills PR, Williams GL. Anatomy of root canals. III. Permanent mandibular molars. Aust Dent J 1974;19:403-13.

2. Vertucci FJ. Root canal anatomy of human permanent teeth. Oral Surg Oral Med Oral Pathol 1984;58:589-99.

3. Van Velzen SK, Wesselink PR, De Cleen MJ. Endodontologie. $2^{\text {nd }}$ ed. Houtem/Diegem: Bohn Stafleu Van Loghum; 1995. p. $142-3$.

4. Fabra-Campos H. Unusual root anatomy of mandibular first molars. J Endod 1985;11:568-57.

5. Fabra-Campos H. Three canals in mesial root of mandibular first permanent molars: A clinical study. Int Endod J 1989; 22:39-43.

6. Bond JL. Clinical management of middle mesial root canals in mandibular molars. J Endod 1988; 14:312-4

7. Stoner WF. Mandibular first molar with three distal canals. Oral Surg 1984; 57:554-7.

8. Carabelli G. Systematisches Handbuch der Zahnheilkunde. 2 ed. Vienna, Italy: Braumuller und Seidel; 1844. p. 114.
9. Carlsen O,Alexandersen V. Radix entomolaris: Identification and morphology. Scand J Dent Res 1990;98:363-73.

10. Garg AK, Tewari RK, Kumar A, Hashmi SH, Agarwal N, Mishra SK. Prevalence of three-rooted mandibular permanent first molars among the Indian population. J Endod 2010;36:1302-6.

11. Calberson FL, De Moor RJ, Deroose CA. The radix entomolaris and paramolaris: Clinical approach in endodontics. J Endod 2007;33:58-63.

12. Curzon ME, Curzon JA. Three rooted mandibular molars in the Keewatin Eskimo. J Can Dent Assoc (Tor) 1971;37:71-2.

13. Walker RT. Root form and canal anatomy of mandibular second molars in a Southern Chinese population. Endod DentTraumatol 1988; 14:325-29.

14. Reichart PA, Metah D. Three rooted permanent mandibular first molars in Thai. Community Dent Oral Epidemiol $1981 ; 9: 191-2$

15. Rebeiro FC, Consolaro A. Importancia clinicia y antropologica de la raiz distolingual en los molars inferiores permanents. Endodontia 1997;15:72-8.

16. Albella F, Patel S, Duran-Sindreu F, Mercade M, Roig M. Mandibular first molars with disto-lingual roots: Review and clinical management. Int Endod J 2012;45:963-78.

17. Tu MG, Huang HL, Hsue SS, Hsu JT, Chey SY, Jou MJ, et al. Detection of permanent three-rooted mandibular first molars by cone-beam computed tomography imaging in Taiwanese individuals. J Endod 2009; 35:503-7.

18. Tu MG, Tsai CC, Jou MJ, Chen WL, Chang YF, Chen SY, et al. Prevalence of three-rooted mandibular first molars among Taiwanese individuals. J Endod 2007;33:1163-6.

19. Bolk L. Which series of the teeth belongs to molars? Z Morphol Anthropol 1914;17:83-116.

20. Visser JB. Beitrag zur Kenntnis der Menschlichen Zahnwurzelformen. Hilversum: Rotting; 1948. p. 49-72.

21. De Moor RJ, Deroose CA, Calberson FL. The radix entomolaris in mandibular molars: An endodontic challenge. Int Endod J 2004;37:789-99.

22. Song JS, Choi HJ, Jung IY, Jung HS, Kim SO. The prevalence and morphologic classification of distolingual roots in the mandibular molars in a Korean population. J Endod 2010;36:653-7.

23. Klein RM, Blake SA, Nattress BR, Hirschmann PN Evaluation of X-ray beam angulation for successful twin canal identification in mandibular incisors. Int Endod J 1997;30:58-63. 